\section{SREBPs: activators of the complete program of cholesterol and fatty acid synthesis in the liver}

\author{
Jay D. Horton, ${ }^{1,2}$ Joseph L. Goldstein, ${ }^{1}$ and \\ Michael S. Brown ${ }^{1}$ \\ ${ }^{1}$ Department of Molecular Genetics, and \\ ${ }^{2}$ Department of Internal Medicine, University of Texas Southwestern \\ Medical Center, Dallas, Texas, USA \\ Address correspondence to: Jay D. Horton, Department of Molecular \\ Genetics, University of Texas Southwestern Medical Center, \\ 5323 Harry Hines Boulevard, Room L5.238, Dallas, Texas 73590-9046, \\ USA. Phone: (214) 648-9677; Fax: (214) 648-8804; \\ E-mail: jay.horton@utsouthwestern.edu. \\ J. Clin. Invest. 109:1125-1131 (2002). DOI:10.1172/JCI200215593.
}

Lipid homeostasis in vertebrate cells is regulated by a family of membrane-bound transcription factors designated sterol regulatory element-binding proteins (SREBPs). SREBPs directly activate the expression of more than 30 genes dedicated to the synthesis and uptake of cholesterol, fatty acids, triglycerides, and phospholipids, as well as the NADPH cofactor required to synthesize these molecules (1-4). In the liver, three SREBPs regulate the production of lipids for export into the plasma as lipoproteins and into the bile as micelles. The complex, interdigitated roles of these three SREBPs have been dissected through the study of ten different lines of gene-manipulated mice. These studies form the subject of this review.

\section{SREBPs: activation through proteolytic processing}

SREBPs belong to the basic helix-loop-helix-leucine zipper (bHLH-Zip) family of transcription factors, but they differ from other bHLH-Zip proteins in that they are synthesized as inactive precursors bound to the endoplasmic reticulum $(E R)(1,5)$. Each SREBP precursor of about 1150 amino acids is organized into three domains: (a) an $\mathrm{NH}_{2}$-terminal domain of about 480 amino acids that contains the bHLH-Zip region for binding DNA; (b) two hydrophobic transmembrane-spanning segments interrupted by a short loop of about 30 amino acids that projects into the lumen of the ER; and (c) a COOH-terminal domain of about 590 amino acids that performs the essential regulatory function described below.

In order to reach the nucleus and act as a transcription factor, the $\mathrm{NH}_{2}$-terminal domain of each SREBP must be released from the membrane proteolytically (Figure 1). Three proteins required for SREBP processing have been delineated in cultured cells, using the tools of somatic cell genetics (see ref. 5 for review). One is an escort protein designated SREBP cleavage-activating protein (SCAP). The other two are proteases, designated Site-1 protease (S1P) and Site-2 protease (S2P). Newly synthesized SREBP is inserted into the membranes of the $\mathrm{ER}$, where its $\mathrm{COOH}$-terminal regulatory domain binds to the COOH-terminal domain of SCAP (Figure 1). SCAP is both an escort for SREBPs and a sensor of sterols. When cells become depleted in cholesterol, SCAP escorts the SREBP from the ER to the Golgi apparatus, where the two proteases reside. In the Golgi apparatus, S1P, a membrane-bound serine protease, cleaves the SREBP in the luminal loop between its two membrane-spanning segments, dividing the SREBP molecule in half (Figure 1). The $\mathrm{NH}_{2}$-terminal bHLH$\mathrm{Zip}$ domain is then released from the membrane via a second cleavage mediated by S2P, a membrane-bound zinc metalloproteinase. The $\mathrm{NH}_{2}$-terminal domain, designated nuclear SREBP (nSREBP), translocates to the nucleus, where it activates transcription by binding to nonpalindromic sterol response elements (SREs) in the promoter/enhancer regions of multiple target genes.

When the cholesterol content of cells rises, SCAP senses the excess cholesterol through its membranous sterol-sensing domain, changing its conformation in such a way that the SCAP/SREBP complex is no longer incorporated into ER transport vesicles. The net result is that SREBPs lose their access to S1P and S2P in the Golgi apparatus, so their bHLH-Zip domains cannot be released from the ER membrane, and the transcription of target genes ceases $(1,5)$. The biophysical mechanism by which SCAP senses sterol levels in the ER membrane and regulates its movement to the Golgi apparatus is not yet understood. Elucidating this mechanism will be fundamental to understanding the molecular basis of cholesterol feedback inhibition of gene expression.

\section{SREBPs: two genes, three proteins}

The mammalian genome encodes three SREBP isoforms, designated SREBP-1a, SREBP-1c, and SREBP-2. SREBP-2 is encoded by a gene on human chromosome 22q13. Both SREBP-1a and -1c are derived from a single gene on human chromosome 17p11.2 through the use of alternative transcription start sites that produce alternate forms of exon 1, designated 1a and 1c (1). SREBP-1a is a potent activator of all SREBP-responsive genes, including those that mediate the synthesis of cholesterol, fatty acids, and triglycerides. High-level transcriptional activation is dependent on exon 1a, which encodes a longer acidic transactivation segment than does the first exon of SREBP-1c. The roles of SREBP-1c and SREBP-2 are more restricted than that of SREBP-1a. SREBP-1c preferentially enhances transcription of genes required for fatty acid synthesis but not cholesterol syn- 


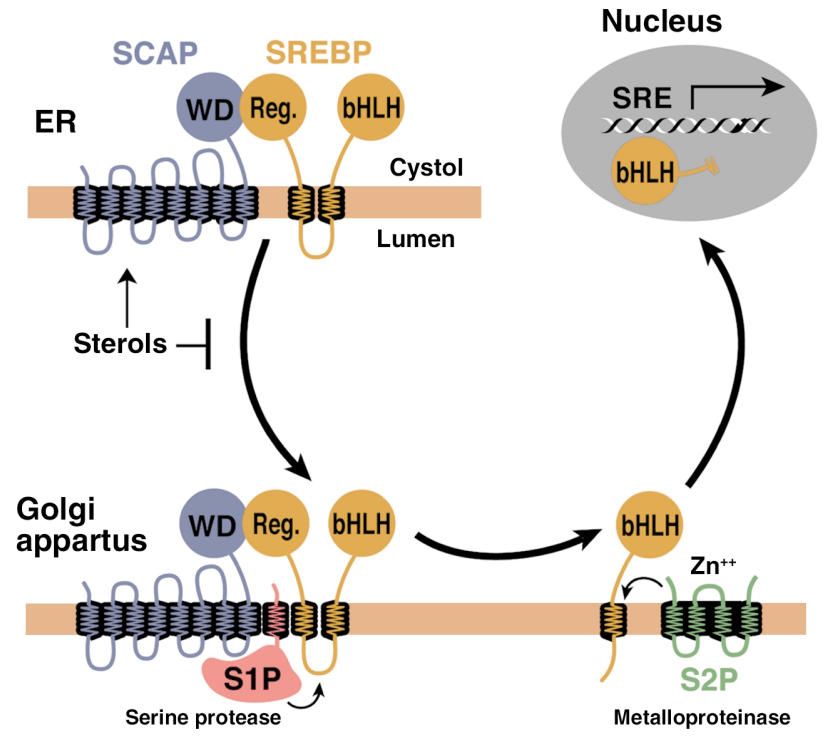

Figure 1

Model for the sterol-mediated proteolytic release of SREBPs from membranes. SCAP is a sensor of sterols and an escort of SREBPs. When cells are depleted of sterols, SCAP transports SREBPs from the ER to the Golgi apparatus, where two proteases, Site-1 protease (S1P) and Site- 2 protease (S2P), act sequentially to release the $\mathrm{NH}_{2}$-terminal bHLH-Zip domain from the membrane. The bHLH-Zip domain enters the nucleus and binds to a sterol response element (SRE) in the enhancer/promoter region of target genes, activating their transcription. When cellular cholesterol rises, the SCAP/SREBP complex is no longer incorporated into ER transport vesicles, SREBPs no longer reach the Golgi apparatus, and the bHLH-Zip domain cannot be released from the membrane. As a result, transcription of all target genes declines. Reprinted from ref. 5 with permission.

thesis. Like SREBP-1a, SREBP-2 has a long transcriptional activation domain, but it preferentially activates cholesterol synthesis (1). SREBP-1a and SREBP-2 are the predominant isoforms of SREBP in most cultured cell lines, whereas SREBP-1c and SREBP-2 predominate in the liver and most other intact tissues (6).

When expressed at higher than physiologic levels, each of the three SREBP isoforms can activate all enzymes indicated in Figure 2, which shows the biosynthetic pathways used to generate cholesterol and fatty acids. However, at normal levels of expression, SREBP-1c favors the fatty acid biosynthetic pathway and SREBP-2 favors cholesterologenesis. SREBP2 -responsive genes in the cholesterol biosynthetic pathway include those for the enzymes HMG-CoA synthase, HMG-CoA reductase, farnesyl diphosphate synthase, and squalene synthase. SREBP-1c-responsive genes include those for ATP citrate lyase (which produces acetyl-CoA) and acetyl-CoA carboxylase and fatty acid synthase (which together produce palmitate [C16:0]). Other SREBP-1c target genes encode a ratelimiting enzyme of the fatty acid elongase complex, which converts palmitate to stearate (C18:0) (ref. 7); stearoyl-CoA desaturase, which converts stearate to oleate (C18:1); and glycerol-3-phosphate acyltransferase, the first committed enzyme in triglyceride and phospholipid synthesis (3). Finally, SREBP-1c and SREBP-2 activate three genes required to generate
$\mathrm{NADPH}$, which is consumed at multiple stages in these lipid biosynthetic pathways (8) (Figure 2).

\section{Knockout and transgenic mice}

Ten different genetically manipulated mouse models that either lack or overexpress a single component of the SREBP pathway have been generated in the last 6 years (9-16). The key molecular and metabolic alterations observed in these mice are summarized in Table 1.

Knockout mice that lack all nSREBPs die early in embryonic development. For instance, a germline deletion of S1p, which prevents the processing of all SREBP isoforms, results in death before day 4 of development $(15,17)$. Germline deletion of Srebp2 leads to $100 \%$ lethality at a later stage of embryonic development than does deletion of S1p (embryonic day 7-8). In contrast, germline deletion of Srebp1, which eliminates both the $1 \mathrm{a}$ and the $1 \mathrm{c}$ transcripts, leads to partial lethality, in that about $15-45 \%$ of Srebp $1^{-/-}$mice survive (13). The surviving homozygotes manifest elevated levels of SREBP- 2 mRNA and protein (Table 1), which presumably compensates for the loss of SREBP- $1 \mathrm{a}$ and -1c. When the SREBP-1c transcript is selectively eliminated, no embryonic lethality is observed, suggesting that the partial embryonic lethality in the Srebp $1^{-/-}$mice is due to the loss of the SREBP-1a transcript (16).

To bypass embryonic lethality, we have produced mice in which all SREBP function can be disrupted in adulthood through induction of Cre recombinase. For this purpose, loxP recombination sites were inserted into genomic regions that flank crucial exons in the Scap or S1p genes (so-called floxed alleles) $(14,15)$. Mice homozygous for the floxed gene and heterozygous for a Cre recombinase transgene, which is under control of an IFN-inducible promoter (MX1-Cre), can be induced to delete Scap or S1p by stimulating IFN expression. Thus, following injection with polyinosinic acid-polycytidylic acid, a double-stranded RNA that provokes antiviral responses, the Cre recombinase is produced in liver and disrupts the floxed gene by recombination between the loxP sites.

Cre-mediated disruption of Scap or S1p dramatically reduces nSREBP-1 and nSREBP-2 levels in liver and diminishes expression of all SREBP target genes in both the cholesterol and the fatty acid synthetic pathways (Table 1). As a result, the rates of synthesis of cholesterol and fatty acids fall by $70-80 \%$ in Scap- and S1p-deficient livers.

In cultured cells, the processing of SREBP is inhibited by sterols, and the sensor for this inhibition is SCAP (5). To learn whether SCAP performs the same function in liver, we have produced transgenic mice that express a mutant SCAP with a single amino acid substitution in the sterol-sensing domain (D443N) (12). Studies in tissue culture show that $\mathrm{SCAP}(\mathrm{D} 443 \mathrm{~N})$ is resistant to inhibition by sterols. Cells that express a single copy of this mutant gene overproduce cholesterol (18). Transgenic mice that express this mutant version of SCAP in the liver exhibit a similar phenotype (12). These livers manifest elevated levels of nSREBP-1 and nSREBP-2, owing to constitutive SREBP processing, which is not suppressed when the animals are fed a cholesterol-rich 
Table 1

Alterations in hepatic lipid metabolism in gene-manipulated mice overexpressing or lacking SREBPs

\begin{tabular}{lccccc}
\hline $\begin{array}{l}\text { Genetic } \\
\text { manipulation }\end{array}$ & $\begin{array}{c}\text { Amount of } \\
\text { nSREBPs }\end{array}$ & $\begin{array}{c}\text { Expression of } \\
\text { target genes }\end{array}$ & Lipid & Liver & Plasma \\
synthesis & content & levels \\
& & HMGR FAS LDLR & Chol FA & Chol TG & Chol TG
\end{tabular}

Fold difference relative to values in wild-type mice

\begin{tabular}{|c|c|c|c|c|c|c|c|c|c|}
\hline \multicolumn{10}{|l|}{ Transgenic mice } \\
\hline SREBP-1a $(9,11)$ & $\uparrow 1 \mathrm{a}$ & $\uparrow 37$ & $\uparrow 20$ & $\uparrow 6$ & $\uparrow 5$ & $\uparrow 26$ & $\uparrow 6$ & $\uparrow 22$ & $\downarrow 0.7$ \\
\hline SREBP-1c $(10,11)$ & $\uparrow 1 c$ & n.c. & $\uparrow 4$ & n.c. & n.c. & $\uparrow 4$ & n.c. & $\uparrow 4$ & n.c. \\
\hline SREBP-2 (11) & $\uparrow 2$ & $\uparrow 75$ & $\uparrow 15$ & $\uparrow 6$ & $\uparrow 28$ & $\uparrow 4$ & $\uparrow 3$ & $\uparrow 4$ & n.c. \\
\hline $\operatorname{SCAP}(\mathrm{D} 443 \mathrm{~N})(12)$ & $\uparrow 1 \mathrm{a}, \uparrow 1 \mathrm{c}, \uparrow 2$ & $\uparrow 18$ & $\uparrow 11$ & $\uparrow 2$ & $\uparrow 5$ & $\uparrow 7$ & $\uparrow 6$ & $\uparrow 9$ & $\downarrow 0.5$ \\
\hline \multicolumn{10}{|c|}{ Liver-specific knockout mice } \\
\hline $\operatorname{SCAP}(14)$ & $\downarrow_{1 \mathrm{a},} \downarrow_{1 \mathrm{c},} \downarrow_{2}$ & $\downarrow 0.1$ & $\downarrow 0.1$ & $\downarrow 0.3$ & $\downarrow 0.3$ & $\downarrow 0.2$ & $\downarrow 0.8$ & $\downarrow 0.4$ & $\downarrow 0.8$ \\
\hline $\mathrm{S} 1 \mathrm{P}(15)$ & 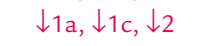 & $\downarrow_{0.6}$ & $\downarrow 0.3$ & $\downarrow 0.5$ & $\downarrow 0.3$ & $\downarrow 0.3$ & n.c. & $\downarrow 0.5$ & $\downarrow 0.6$ \\
\hline \multicolumn{10}{|c|}{ Germline knockout mice } \\
\hline SREBP-1a \& 1c (13) & $\downarrow_{1 \mathrm{a},} \downarrow_{1 \mathrm{c},} \uparrow 2$ & $\uparrow 2.2$ & $\downarrow 0.7$ & $\uparrow 1.3$ & $\uparrow 3$ & $\downarrow 0.6$ & $\uparrow 1.5$ & n.c & $\downarrow 0.7$ \\
\hline SREBP-1c (16) & $\downarrow 1 \mathrm{a}, \downarrow 1 \mathrm{c}, \uparrow 2$ & $\uparrow 1.3$ & $\downarrow 0.3$ & n.c & $\uparrow 3$ & $\downarrow 0.5$ & $\uparrow 1.2$ & n.c & $\downarrow 0.8$ \\
\hline SREBP-2 (13) & Embryonic lethal & - & - & - & - & - & - & - & - \\
\hline $\operatorname{S} 1 \mathrm{P}(15,17)$ & Embryonic lethal & - & - & - & - & - & - & - & - \\
\hline
\end{tabular}

HMGR, HMG-CoA reductase; FAS, fatty acid synthase; LDLR, LDL receptor; Chol, cholesterol; TG, triglycerides; n.c., no change.

diet. nSREBP-1 and -2 increase the expression of all SREBP target genes shown in Figure 2, thus stimulating cholesterol and fatty acid synthesis and causing a marked accumulation of hepatic cholesterol and triglycerides (Table 1). This transgenic model provides strong in vivo evidence that SCAP activity is normally under partial inhibition by endogenous sterols, which keeps the synthesis of cholesterol and fatty acids in a partially repressed state in the liver.

\section{Function of individual SREBP isoforms in vivo}

To study the functions of individual SREBPs in the liver, we have produced transgenic mice that overexpress truncated versions of SREBPs (nSREBPs) that terminate prior to the membrane attachment domain. These nSREBPs enter the nucleus directly, bypassing the sterol-regulated cleavage step. By studying each nSREBP isoform separately, we could determine their distinct activating properties, albeit when overexpressed at nonphysiologic levels.

Overexpression of nSREBP-1c in the liver of transgenic mice produces a triglyceride-enriched fatty liver with no increase in cholesterol (10). mRNAs for fatty acid synthetic enzymes and rates of fatty acid synthesis are elevated fourfold in this tissue, whereas the mRNAs for cholesterol synthetic enzymes and the rate of cholesterol synthesis are not increased (8). Conversely, overexpression of nSREBP-2 in the liver increases the mRNAs encoding all cholesterol biosynthetic enzymes; the most dramatic is a 75-fold increase in HMG-CoA reductase mRNA (11). mRNAs for fatty acid synthesis enzymes are increased to a lesser extent, consistent with the in vivo observation that the rate of cholesterol synthesis increases 28 -fold in these transgenic nSREBP-2 livers, while fatty acid synthesis increases only fourfold. This increase in cholesterol synthesis is even more remarkable when one considers the extent of cholesterol overload in this tissue, which would ordinarily reduce SREBP processing and essentially abolish cholesterol synthesis (Table 1).

We have also studied the consequences of overexpressing SREBP-1a, which is expressed only at low levels in the livers of adult mice, rats, hamsters, and humans (6). nSREBP-1a transgenic mice develop a massive fatty liver engorged with both cholesterol and triglycerides (9), with heightened expression of genes controlling cholesterol biosynthesis and, still more dramatically, fatty acid synthesis (Table 1 ). The preferential activation of fatty acid synthesis (26-fold increase) relative to cholesterol synthesis (fivefold increase) explains the greater accumulation of triglycerides in their livers. The relative representation of the various fatty acids accumulating in this tissue is also unusual. Transgenic nSREBP-1a livers contain about $65 \%$ oleate (C18:1), markedly higher levels than the $15-20 \%$ found in typical wild-type livers (8) - a result of the induction of fatty acid elongase and stearoyl-CoA desaturase- 1 (7). Considered together, the overexpression studies indicate that both SREBP-1 isoforms show a relative preference for activating fatty acid synthesis, whereas SREBP-2 favors cholesterol.

The phenotype of animals lacking the Srebp1 gene, which encodes both the SREBP-1a and -1c transcripts, also supports the notion of distinct hepatic functions for SREBP-1 and SREBP-2 (13). Most homozygous SREBP-1 knockout mice die in utero. The surviving Srebp1 $1^{-/}$mice show reduced synthesis of fatty acids, owing to reduced expression of mRNAs for fatty acid synthetic enzymes (Table 1). Hepatic nSREBP-2 levels increase in these mice, presumably in compensation for the loss of nSREBP-1. As a result, transcription of cholesterol biosynthetic genes increases, producing a threefold increase in hepatic cholesterol synthesis (Table 1 ).

The studies in genetically manipulated mice clearly show that, as in cultured cells, SCAP and S1P are 


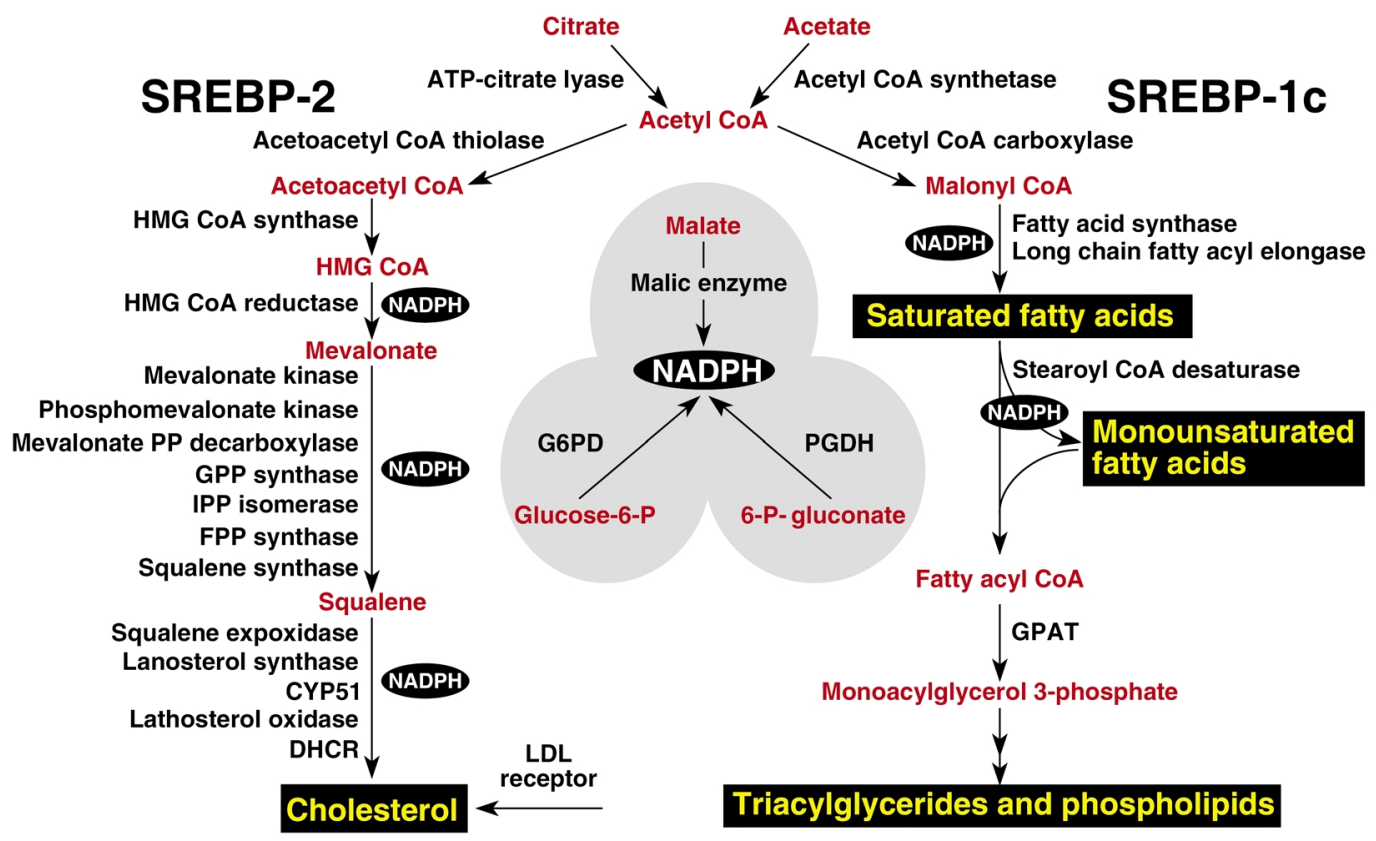

\section{Figure 2}

Genes regulated by SREBPs. The diagram shows the major metabolic intermediates in the pathways for synthesis of cholesterol, fatty acids, and triglycerides. In vivo, SREBP-2 preferentially activates genes of cholesterol metabolism, whereas SREBP-1c preferentially activates genes of fatty acid and triglyceride metabolism. DHCR, 7-dehydrocholesterol reductase; FPP, farnesyl diphosphate; GPP, geranylgeranyl pyrophosphate synthase; CYP51, lanosterol 14 $\alpha$-demethylase; G6PD, glucose-6-phosphate dehydrogenase; PGDH, 6-phosphogluconate dehydrogenase; GPAT, glycerol-3-phosphate acyltransferase.

required for normal SREBP processing in the liver. SCAP, acting through its sterol-sensing domain, mediates feedback regulation of cholesterol synthesis. The SREBPs play related but distinct roles: SREBP-1c, the predominant SREBP-1 isoform in adult liver, preferentially activates genes required for fatty acid synthesis, while SREBP-2 preferentially activates the LDL receptor gene and various genes required for cholesterol synthesis. SREBP-1a and SREBP-2, but not SREBP-1c, are required for normal embryogenesis.

\section{Transcriptional regulation of SREBP genes}

Regulation of SREBPs occurs at two levels - transcriptional and posttranscriptional. The posttranscriptional regulation discussed above involves the sterol-mediated suppression of SREBP cleavage, which results from sterol-mediated suppression of the movement of the SCAP/SREBP complex from the ER to the Golgi apparatus (Figure 1). This form of regulation is manifest not only in cultured cells (1), but also in the livers of rodents fed cholesterol-enriched diets (19).

The transcriptional regulation of the SREBPs is more complex. SREBP-1c and SREBP-2 are subject to distinct forms of transcriptional regulation, whereas SREBP-1a appears to be constitutively expressed at low levels in liver and most other tissues of adult animals (6). One mechanism of regulation shared by SREBP-1c and SREBP-2 involves a feed-forward regulation mediated by SREs present in the enhancer/promoters of each gene $(20,21)$. Through this feed-forward loop, nSREBPs activate the transcription of their own genes. In contrast, when nSREBPs decline, as in Scap or S1p knockout mice, there is a secondary decline in the mRNAs encoding SREBP-1c and SREBP- $2(14,15)$.

Three factors selectively regulate the transcription of SREBP-1c: liver X-activated receptors (LXRs), insulin, and glucagon. LXR $\alpha$ and LXR $\beta$, nuclear receptors that form heterodimers with retinoid $X$ receptors, are activated by a variety of sterols, including oxysterol intermediates that form during cholesterol biosynthesis (22-24). An LXR-binding site in the SREBP-1c promoter activates SREBP-1c transcription in the presence of LXR agonists (23). The functional significance of LXR-mediated SREBP-1c regulation has been confirmed in two animal models. Mice that lack both LXR $\alpha$ and LXR $\beta$ express reduced levels of SREBP-1c and its lipogenic target enzymes in liver and respond relatively weakly to treatment with a synthetic LXR agonist (23). Because a similar blunted response is found in mice that lack SREBP-1c, it appears that LXR increases fatty acid synthesis largely by inducing SREBP-1c (16). LXR-mediated activation of SREBP-1c transcription provides a mechanism for the cell to induce the synthesis of oleate when sterols are in excess (23). Oleate is the preferred fatty acid for the synthesis of cholesteryl esters, which are necessary for both the transport and the storage of cholesterol.

LXR-mediated regulation of SREBP-1c appears also to be one mechanism by which unsaturated fatty acids suppress SREBP-1c transcription and thus fatty acid synthesis. Rodents fed diets enriched in polyunsaturated fatty acids manifest reduced SREBP-1c mRNA expression and low rates of lipogenesis in liver (25). In vitro, unsaturated fatty acids competitively block LXR 
activation of SREBP-1c expression by antagonizing the activation of LXR by its endogenous ligands (26). In addition to LXR-mediated transcriptional inhibition, polyunsaturated fatty acids lower SREBP-1c levels by accelerating degradation of its mRNA (27). These combined effects may contribute to the longrecognized ability of polyunsaturated fatty acids to lower plasma triglyceride levels.

\section{SREBP-1 $c$ and the insulin/glucagon ratio}

The liver is the organ responsible for the conversion of excess carbohydrates to fatty acids to be stored as triglycerides or burned in muscle. A classic action of insulin is to stimulate fatty acid synthesis in liver during times of carbohydrate excess. The action of insulin is opposed by glucagon, which acts by raising cAMP. Multiple lines of evidence suggest that insulin's stimulatory effect on fatty acid synthesis is mediated by an increase in SREBP-1c. In isolated rat hepatocytes, insulin treatment increases the amount of mRNA for SREBP-1c in parallel with the mRNAs of its target genes $(28,29)$. The induction of the target genes can be blocked if a dominant negative form of SREBP-1c is expressed (30). Conversely, incubating primary hepatocytes with glucagon or dibutyryl cAMP decreases the mRNAs for SREBP-1c and its associated lipogenic target genes $(30,31)$.

In vivo, the total amount of SREBP-1c in liver and adipose tissue is reduced by fasting, which suppresses insulin and increases glucagon levels, and is elevated by refeeding $(32,33)$. The levels of mRNA for SREBP-1c target genes parallel the changes in SREBP-1c expression. Similarly, SREBP-1c mRNA levels fall when rats are treated with streptozotocin, which abolishes insulin secretion, and rise after insulin injection (29). Overexpression of nSREBP- $1 c$ in livers of transgenic mice prevents the reduction in lipogenic mRNAs that normally follows a fall in plasma insulin levels (32). Conversely, in livers of Scap knockout mice that lack all nSREBPs in the liver (14) or knockout mice lacking either nSREBP-1c (16) or both SREBP-1 isoforms (34), there is a marked decrease in the insulin-induced stimulation of lipogenic gene expression that normally occurs after fasting/refeeding. It should be noted that insulin and glucagon also exert a posttranslational control of fatty acid synthesis though changes in the phosphorylation and activation of acetyl-CoA carboxylase. The posttranslational regulation of fatty acid synthesis persists in transgenic mice that overexpress nSREBP-1c (10). In these mice, the rates of fatty acid synthesis, as measured by $\left[{ }^{3} \mathrm{H}\right]$ water incorporation, decline after fasting even though the levels of the lipogenic mRNAs remain high (our unpublished observations).

Taken together, the above evidence suggests that SREBP-1c mediates insulin's lipogenic actions in liver. Recent in vitro and in vivo studies involving adenoviral gene transfer suggest that SREBP-1c may also contribute to the regulation of glucose uptake and glucose synthesis. When overexpressed in hepatocytes, nSREBP-1c induces expression of glucokinase, a key enzyme in glucose utilization. It also suppresses phosphoenolpyruvate carboxykinase, a key gluconeogenic enzyme $(35,36)$.

\section{SREBPs in disease}

Many individuals with obesity and insulin resistance also have fatty livers, one of the most commonly encountered liver abnormalities in the US (37). A subset of individuals with fatty liver go on to develop fibrosis, cirrhosis, and liver failure. Evidence indicates that the fatty liver of insulin resistance is caused by SREBP-1c, which is elevated in response to the high insulin levels. Thus, SREBP-1c levels are elevated in the fatty livers of obese $(o b / o b)$ mice with insulin resistance and hyperinsulinemia caused by leptin deficiency $(38$, 39). Despite the presence of insulin resistance in peripheral tissues, insulin continues to activate SREBP-1c transcription and cleavage in the livers of these insulin-resistant mice. The elevated nSREBP-1c increases lipogenic gene expression, enhances fatty acid synthesis, and accelerates triglyceride accumulation $(31,39)$. These metabolic abnormalities are reversed with the administration of leptin, which corrects the insulin resistance and lowers the insulin levels (38).

Metformin, a biguanide drug used to treat insulinresistant diabetes, reduces hepatic nSREBP-1 levels and dramatically lowers the lipid accumulation in livers of insulin-resistant $o b / o b$ mice (40). Metformin stimulates AMP-activated protein kinase (AMPK), an enzyme that inhibits lipid synthesis through phosphorylation and inactivation of key lipogenic enzymes (41). In rat hepatocytes, metformin-induced activation of AMPK also leads to decreased mRNA expression of SREBP-1c and its lipogenic target genes (41), but the basis of this effect is not understood.

The incidence of coronary artery disease increases with increasing plasma LDL-cholesterol levels, which in turn are inversely proportional to the levels of hepatic LDL receptors. SREBPs stimulate LDL receptor expression, but they also enhance lipid synthesis (1), so their net effect on plasma lipoprotein levels depends on a balance between opposing effects. In mice, the plasma levels of lipoproteins tend to fall when SREBPs are either overexpressed or underexpressed. In transgenic mice that overexpress nSREBPs in liver, plasma cholesterol and triglycerides are generally lower than in control mice (Table 1), even though these mice massively overproduce fatty acids, cholesterol, or both. Hepatocytes of nSREBP-1a transgenic mice overproduce VLDL, but these particles are rapidly removed through the action of LDL receptors, and they do not accumulate in the plasma. Indeed, some nascent VLDL particles are degraded even before secretion by a process that is mediated by LDL receptors (42). The high levels of nSREBP- $1 \mathrm{a}$ in these animals support continued expression of the LDL receptor, even in cells whose cholesterol concentration is elevated. In LDL receptor-deficient mice carrying the nSREBP-1a transgene, plasma cholesterol and triglyceride levels rise tenfold (43).

Mice that lack all SREBPs in liver as a result of disruption of Scap or S1p also manifest lower plasma cholesterol and triglyceride levels (Table 1). In these mice, hepatic cholesterol and triglyceride synthesis is markedly reduced, and this likely causes a decrease in VLDL production and secretion. LDL receptor mRNA and LDL clearance from plasma is also significantly 
reduced in these mice, but the reduction in LDL clearance is less than the overall reduction in VLDL secretion, the net result being a decrease in plasma lipid levels (15). However, because humans and mice differ substantially with regard to LDL receptor expression, LDL levels, and other aspects of lipoprotein metabolism, it is difficult to predict whether human plasma lipids will rise or fall when the SREBP pathway is blocked or activated.

\section{SREBPs in liver: unanswered questions}

The studies of SREBPs in liver have exposed a complex regulatory system whose individual parts are coming into focus. Major unanswered questions relate to the ways in which the transcriptional and posttranscriptional controls on SREBP activity are integrated so as to permit independent regulation of cholesterol and fatty acid synthesis in specific nutritional states. A few clues regarding these integration mechanisms are discussed below.

Whereas cholesterol synthesis depends almost entirely on SREBPs, fatty acid synthesis is only partially dependent on these proteins. This has been shown most clearly in cultured nonhepatic cells such as Chinese hamster ovary cells. In the absence of SREBP processing, as when the Site- 2 protease is defective, the levels of mRNAs encoding cholesterol biosynthetic enzymes and the rates of cholesterol synthesis decline nearly to undetectable levels, whereas the rate of fatty acid synthesis is reduced by only $30 \%$ (44). Under these conditions, transcription of the fatty acid biosynthetic genes must be maintained by factors other than SREBPs. In liver, the gene encoding fatty acid synthase $(F A S N)$ can be activated transcriptionally by upstream stimulatory factor, which acts in concert with SREBPs (45). The FASN promoter also contains an LXR element that permits a low-level response to LXR ligands even when SREBPs are suppressed (46). These two transcription factors may help to maintain fatty acid synthesis in liver when nSREBP- $1 \mathrm{c}$ is low.

Another mechanism of differential regulation is seen in the ability of cholesterol to block the processing of SREBP-2, but not SREBP-1, under certain metabolic conditions. This differential regulation has been studied most thoroughly in cultured cells such as human embryonic kidney (HEK-293) cells. When these cells are incubated in the absence of fatty acids and cholesterol, the addition of sterols blocks processing of SREBP-2, but not SREBP-1, which is largely produced as SREBP-1a in these cells (47). Inhibition of SREBP-1 processing requires an unsaturated fatty acid, such as oleate or arachidonate, in addition to sterols (47). In the absence of fatty acids and in the presence of sterols, SCAP may be able to carry SREBP-1 proteins, but not SREBP-2, to the Golgi apparatus. Further studies are necessary to document this apparent independent regulation of SREBP-1 and SREBP-2 processing and to determine its mechanism.

\section{Acknowledgments}

Support for the research cited from the authors' laboratories was provided by grants from the NIH (HL-
20948), the Moss Heart Foundation, the Keck Foundation, and the Perot Family Foundation. J.D. Horton is a Pew Scholar in the Biomedical Sciences and is the recipient of an Established Investigator Grant from the American Heart Association and a Research Scholar Award from the American Digestive Health Industry.

1. Brown, M.S., and Goldstein, J.L. 1997. The SREBP pathway: regulation of cholesterol metabolism by proteolysis of a membrane-bound transcription factor. Cell. 89:331-340.

2. Horton, J.D., and Shimomura, I. 1999. Sterol regulatory element-binding proteins: activators of cholesterol and fatty acid biosynthesis. Curr. Opin. Lipidol. 10:143-150.

3. Edwards, P.A., Tabor, D., Kast, H.R., and Venkateswaran, A. 2000. Regulation of gene expression by SREBP and SCAP. Biochim. Biophys. Acta. 1529:103-113.

4. Sakakura, Y., et al. 2001. Sterol regulatory element-binding proteins induce an entire pathway of cholesterol synthesis. Biochem. Biophys. Res. Comm. 286:176-183.

5. Goldstein, J.L., Rawson, R.B., and Brown, M.S. 2002. Mutant mammalian cells as tools to delineate the sterol regulatory element-binding protein pathway for feedback regulation of lipid synthesis. Arch. Biochem. Biophys. 397:139-148.

6. Shimomura, I., Shimano, H., Horton, J.D., Goldstein, J.L., and Brown, M.S. 1997. Differential expression of exons $1 \mathrm{a}$ and $1 \mathrm{c}$ in mRNAs for sterol regulatory element binding protein- 1 in human and mouse organs and cultured cells. J. Clin. Invest. 99:838-845.

7. Moon, Y.-A., Shah, N.A., Mohapatra, S., Warrington, J.A., and Horton, J.D. 2001. Identification of a mammalian long chain fatty acyl elongase regulated by sterol regulatory element-binding proteins. J. Biol. Chem. 276:45358-45366.

8. Shimomura, I., Shimano, H., Korn, B.S., Bashmakov, Y., and Horton, J.D. 1998. Nuclear sterol regulatory element binding proteins activate genes responsible for entire program of unsaturated fatty acid biosynthesis in transgenic mouse liver. J. Biol. Chem. 273:35299-35306.

9. Shimano, H., et al. 1996. Overproduction of cholesterol and fatty acids causes massive liver enlargement in transgenic mice expressing truncated SREBP-1a. J. Clin. Invest. 98:1575-1584.

10. Shimano, H., et al. 1997. Isoform 1c of sterol regulatory element binding protein is less active than isoform $1 \mathrm{a}$ in livers of transgenic mice and in cultured cells. J. Clin. Invest. 99:846-854.

11. Horton, J.D., et al. 1998. Activation of cholesterol synthesis in preference to fatty acid synthesis in liver and adipose tissue of transgenic mice overproducing sterol regulatory element-binding protein-2. J. Clin. Invest. 101:2331-2339.

12. Korn, B.S., et al. 1998. Blunted feedback suppression of SREBP processing by dietary cholesterol in transgenic mice expressing sterol-resistant SCAP(D443N). J. Clin. Invest. 102:2050-2060.

13. Shimano, H., et al. 1997. Elevated levels of SREBP-2 and cholesterol synthesis in livers of mice homozygous for a targeted disruption of the SREBP-1 gene. J. Clin. Invest. 100:2115-2124.

14. Matsuda, M., et al. 2001. SREBP cleavage-activating protein (SCAP) is required for increased lipid synthesis in liver induced by cholesterol deprivation and insulin elevation. Genes Dev. 15:1206-1216.

15. Yang, J., et al. 2001. Decreased lipid synthesis in livers of mice with disrupted Site-1 protease gene. Proc. Natl. Acad. Sci. USA. 98:13607-13612.

16. Liang, G., et al. 2002. Diminished hepatic response to fasting/refeeding and liver $\mathrm{X}$ receptor agonists in mice with selective deficiency of sterol regulatory element-binding protein-1c. J. Biol. Chem. 277:9520-9528.

17. Mitchell, K.J., et al. 2001. Functional analysis of secreted and transmembrane proteins critical to mouse development. Nat. Genet. 28:241-249.

18. Hua, X., Nohturfft, A., Goldstein, J.L., and Brown, M.S. 1996. Sterol resistance in $\mathrm{CHO}$ cells traced to point mutation in SREBP cleavage activating protein (SCAP). Cell. 87:415-426.

19. Shimomura, I., et al. 1997. Cholesterol feeding reduces nuclear forms of sterol regulatory element binding proteins in hamster liver. Proc. Natl. Acad. Sci. USA. 94:12354-12359.

20. Sato, R., et al. 1996. Sterol-dependent transcriptional regulation of sterol regulatory element-binding protein-2. J. Biol. Chem. 271:26461-26464.

21. Amemiya-Kudo, M., et al. 2000. Promoter analysis of the mouse sterol regulatory element-binding protein-1c gene. J. Biol. Chem. 275:31078-31085.

22. Janowski, B.A., et al. 1999. Structural requirements of ligands for the oxysterol liver X receptors LXR $\alpha$ and LXR $\beta$. Proc. Natl. Acad. Sci. USA. 96:266-271.

23. Repa, J.J., et al. 2000. Regulation of mouse sterol regulatory elementbinding protein-1c gene (SREBP-1c) by oxysterol receptors, LXR $\alpha$ and LXRß. Genes Dev. 14:2819-2830.

24. DeBose-Boyd, R.A., Ou, J., Goldstein, J.L., and Brown, M.S. 2001. Expres- 
sion of sterol regulatory element-binding protein 1c (SREBP-1c) mRNA in rat hepatoma cells requires endogenous LXR ligands. Proc. Natl. Acad. Sci. USA. 98:1477-1482.

25. Xu, J., Nakamura, M.T., Cho, H.P., and Clarke, S.D. 1999. Sterol regulatory element binding protein-1 expression is suppressed by dietary polyunsaturated fatty acids. J. Biol. Chem. 274:23577-23583.

26. Ou, J., et al. 2001. Unsaturated fatty acids inhibit transcription of the sterol regulatory element-binding protein-1c (SREBP-1c) gene by antagonizing ligand-dependent activation of the LXR. Proc. Natl. Acad. Sci. USA. 98:6027-6032.

27. Xu, J., Teran-Garcia, M., Park, J.H.Y., Nakamura, M.T., and Clarke, S.D 2001. Polyunsaturated fatty acids suppress hepatic sterol regulatory element-binding protein-1 expression by accelerating transcript decay. $J$. Biol. Chem. 276:9800-9807.

28. Foretz, M., Guichard, C., Ferre, P., and Foufelle, F. 1999. Sterol regulatory element binding protein- $1 \mathrm{c}$ is a major mediator of insulin action on the hepatic expression of glucokinase and lipogenesis-related genes. Proc. Natl. Acad. Sci. USA. 96:12737-12742.

29. Shimomura, I., et al. 1999. Insulin selectively increases SREBP-1c mRNA in livers of rats with streptozotocin-induced diabetes. Proc. Natl. Acad. Sci. USA. 96:13656-13661.

30. Foretz, M., et al. 1999. ADD1/SREBP-1c is required in the activation of hepatic lipogenic gene expression by glucose. Mol. Cell. Biol. 19:3760-3768.

31. Shimomura, I., et al. 2000. Decreased IRS-2 and increased SREBP-1c lead to mixed insulin resistance and sensitivity in livers of lipodystrophic and ob/ob mice. Mol. Cell. 6:77-86.

32. Horton, J.D., Bashmakov, Y., Shimomura, I., and Shimano, H. 1998. Regulation of sterol regulatory element binding proteins in livers of fasted and refed mice. Proc. Natl. Acad. Sci. USA. 95:5987-5992.

33. Kim, J.B., et al. 1998. Nutritional and insulin regulation of fatty acid synthetase and leptin gene expression through ADD1/SREBP1. J. Clin. Invest. 101:1-9.

34. Shimano, H., et al. 1999. Sterol regulatory element-binding protein-1 as a key transcription factor for nutritional induction of lipogenic enzyme genes. J. Biol. Chem. 274:35832-35839.

35. Becard, D., et al. 2001. Adenovirus-mediated overexpression of sterol regulatory element binding protein- $1 \mathrm{c}$ mimics insulin effects on hepatic gene expression and glucose homeostasis in diabetic mice. Diabetes. 50:2425-2430.
36. Chakravarty, K., et al. 2001. Sterol regulatory element-binding protein$1 \mathrm{c}$ mimics the negative effect of insulin on phosphoenolpyruvate carboxykinase (GTP) gene transcription. J. Biol. Chem. 276:34816-34823.

37. Marchesini, G., et al. 2001. Nonalcoholic fatty liver disease. Diabetes. 50:1844-1850.

38. Shimomura, I., Hammer, R.E., Ikemoto, S., Brown, M.S., and Goldstein, J.L. 1999. Leptin reverses insulin resistance and diabetes mellitus in mice with congenital lipodystrophy. Nature. 401:73-76.

39. Shimomura, I., Bashmakov, Y., and Horton, J.D. 1999. Increased levels of nuclear SREBP-1c associated with fatty livers in two mouse models of diabetes mellitus. J. Biol. Chem. 274:30028-30032.

40. Lin, H.Z., et al. 2000. Metformin reverses fatty liver disease in obese, leptin-deficient mice. Nat. Med. 6:998-1003.

41. Zhou, G., et al. 2001. Role of AMP-activated protein kinase in mechanism of metformin action. J. Clin. Invest. 108:1167-1174. DOI:10.1172/JCI200113505.

42. Gillian-Daniel, D.L., Bates, P.W., Tebon, A., and Attie, A.D. 2002. Endoplasmic reticulum localization of the low density lipoprotein receptor mediates pre-secretory degradation of apolipoprotein B. Proc. Natl. Acad. Sci. USA. 99:4337-4342.

43. Horton, J.D., Shimano, H., Hamilton, R.L., Brown, M.S., and Goldstein, J.L. 1999. Disruption of LDL receptor gene in transgenic SREBP-1a mice unmasks hyperlipidemia resulting from production of lipid-rich VLDL. J. Clin. Invest. 103:1067-1076.

44. Pai, J., Guryev, O., Brown, M.S., and Goldstein, J.L. 1998. Differential stimulation of cholesterol and unsaturated fatty acid biosynthesis in cells expressing individual nuclear sterol regulatory element binding proteins. J. Biol. Chem. 273:26138-26148.

45. Latasa, M.-J., Moon, Y.S., Kim, K.-H., and Sul, H.S. 2000. Nutritional regulation of the fatty acid synthase promoter in vivo: sterol regulatory element binding protein functions through an upstream region containing a sterol regulatory element. Proc. Natl. Acad. Sci. USA. 97:10619-10624.

46. Joseph, S.B., et al. 2002. Direct and indirect mechanisms for regulation of fatty acid synthase gene expression by LXRs. J. Biol. Chem. 277:11019-11025.

47. Hannah, V.C., Ou, J., Luong, A., Goldstein, J.L., and Brown, M.S. 2001. Unsaturated fatty acids down-regulate SREBP isoforms 1a and 1c by two mechanisms in HEK-293 cells. J. Biol. Chem. 276:4365-4372. 